\title{
THEORETICAL EXPOSITION OF A SINGLE ELECTRON QUANTUM DOT
}

\author{
Wahyu Tri Cahyanto*, Kamsul Abraha ${ }^{\S}$, Pekik Nurwantoro ${ }^{\S}$
}

\begin{abstract}
This paper will review atomic-like phenomena in a semiconductor quantum dot which their size, shape and interactions can be precisely controlled through the use of nanofabrication technology. It has been shown that by confining electrons in three dimensions inside semiconductors, quantum dots can show many of the phenomena observed in atoms and nuclei and so called artificial atom. Because of its controlable system, it is possible to explore the correlation effects in regimes that cannot otherwise be accessed in other physical systems. For simplicity, this work will focus on a circular-shape single electron quantum dot trapped by polar twodimensional harmonic potential in the presence of an external magnetic field. Both Zeeman splitting and spin orbit interaction are neglected in our calculation. The calculation show that Fock-Darwin spectrum will enter Landau regime, where $E_{n}=\left(n+\frac{1}{2}\right) \hbar \omega_{c}$ when cyclotron frequency is much larger than potential confinement
\end{abstract} $\omega_{c}>\omega_{0}$.

Keywords: quantum dot, nanotechnology, electron confinement, artificial atom.

\begin{abstract}
ABSTRAK
Paper ini meninjau fenomena sifat-sifat yang menyerupai atom dalam quantum dot semikonduktor, yang mana ukuran, bentuk, maupun interaksi-interaksi yang ada dapat diatur dengan mudah melalui teknologi nano-fabrikasi. Telah ditunjukkan bahwa dengan mengungkung elektron-elektron ke semua arah dalam ruang di dalam semikonduktor, quantum dot menunjukkan fenomena-fenomena yang juga dapat diamati dalam atom maupun inti atom, sehingga disebut atom buatan. Karena merupakan sistem terkontrol, quantum dot dapat digunakan untuk mengeksplorasi efek-efek korelasi yang tidak dapat diberikan oleh sistem fisis lain. Untuk penyederhanaan, paper ini hanya fokus pada quantum dot berbentuk lingkaran yang berisi satu elektron dan terkungkung pada potensial harmonik dua dimensi dalam pengaruh medan magnet luar. Efek pemecahan Zeeman maupun interaksi spin orbit diabaikan dalam perhitungan ini. Perhitungan menunjukkan bahwa spektrum Fock-Darwin akan memasuki aras-aras Landau, yaitu $E_{n}=\left(n+\frac{1}{2}\right) \hbar \omega_{c}$ jika frekuensi siklotron jauh lebih besar daripada frekuensi yang terkait dengan potensial pengungkung $\omega_{c}>\omega_{0}$.
\end{abstract}

Kata kunci: quantum dot, nanoteknologi, pengungkungan elektron, atom buatan.

\section{INTRODUCTION}

Semiconductor technology has come along in leaps and bounds since the development of the transistor in 1948. The major trend of the development of electronic components is to improve fabrication methods so that the size of the components becomes smaller and more circuits can be included on a chip. Smaller components also work with smaller currents and voltages and therefore more efficiently with less energy consumption. This progress in semiconductor material fabrication has given scientists the opportunity to explore a new world between the macroscopic world which obeys classical physics and the microscopic world which obeys quantum mechanics. This new world which has the range of nanometers (then called mesoscopics) offers a large variety of interesting phenomena.
The growth of nanotechnology is started with the Thouless idea (Thouless 1977) about possibility to reduce the conductor dimension to the size of just few atoms. This question was replied immediately by the growth of microelectronics fabrication which has succeeded to make a controlled system which is only consisted of just few particles. The restriction particle into two dimensions is known as a quantum well, then the advanced confinement in one dimension is referred to a quantum wire, and electron confinement into all three spatial directions is known as a quantum dot.

Quantum dots are usually made by restricting the two dimensional electron gas (2DEG) in the semiconductor heterostructure laterally using a very small electrostatic gate or vertically by etching technique. Gallium Arsenide (GaAs)

\footnotetext{
* Jurusan Fisika, FMIPA UGM, Yogyakarta (55281)

E-mail: tricahyanto@gmail.com

$\mathbb{S}$ Jurusan Fisika, Program Sarjana, MIPA UNSOED, Purwokerto (53123)
} 
and Alumunium Gallium Arsenide $\left(\mathrm{Al}_{x} \mathrm{Ga}_{1-\mathrm{x}} \mathrm{As}\right)$ are semiconductors with similar lattice constants $(\approx 5.65 \AA$ for $\mathrm{GaAs})$ and thus can be brought together to form a heterostructure. At room temperature the bandgap for $\mathrm{GaAs}$ is $1.424 \mathrm{eV}$, while the bandgap in AlGaAs depends on the mixing factor $\mathrm{x}$ and can be approximated by (Palmer 2003)

$$
E_{g}(x)=1.424+1.429 x-0.14 x^{2}[e V] \quad 0<x<0.44
$$

i.e., it varies between $1.424-2.026 \mathrm{eV}$, leads to a discontinuity at their interface. Working with the GaAs-AlGaAs mentioned above, a twodimensional electron system is typically created by $n$-doping the AlGaAs in a heterostructure (Figure 1).

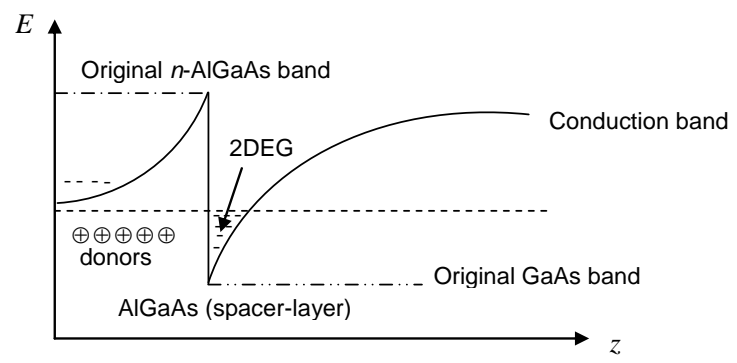

Figure 1.Two-dimensional electron gas seen along the confining dimension.

By doping $\mathrm{Al}_{x} \mathrm{Ga}_{1-\mathrm{x}} \mathrm{As}$-layer with Si-donors, the conduction electrons move to the GaAslayer, since it has a lower bandedge. Some of the electrons added will eventually migrate into the GaAs. These electrons will still be attracted by the positive donors in the $\mathrm{AlGaAs}$, but be unable to go back across the heterojunction because of the conduction band discontinuity. Squeezed in between the discontinuity and the donor potential, they are trapped in a narrow potential well (see Fig 1) and their energy component in this dimension will be quantized. At sufficiently low temperatures all electrons will be in the same (the lowest) energy state with respect to motion perpendicular to the interface. In other word, electrons are free to move in the plane parallel to the heterojunction, but restricted to the same (lowest) energy state in the third dimension.

This paper is organized as follows. In Section 1, a brief review of quantum dot including history and fabrication method are reviewed. Comparison between quantum dot and real atom is given in Section 2. Section 3 will present the model of quantum dot. A quantum dot trapped in 2D polar harmonic oscillator potential in the absence of external magnetic field is analyzed. Shell structure of this quantum dot model also given in Section 3. In
Section 4, analytical results for a single electron quantum dot in the influence of external magnetic field perpendicular to the dot are derived. Finally, Section 5 and 6 give a summary and further research to be done respectively.

\section{QUANTUM DOT VS REAL ATOM}

The interesting features of quantum dots are that they show similar physical properties which we have already seen in atomic physics, then they are called artificial atom. Discretization energy levels, Shell filling, the Zeeman effect, Kondo effect can be found in both real atom and semiconductor quantum dot. In atomic physics, the chemical inertness and special stability of noble gases are explained by a mean-field approach that describes the motion of electrons bound to the three-dimensional spherically symmetric Coulomb potential of the nucleus. This potential around the atoms gives rise to the shell structure $1 s, 2 s, 2 p, \ldots$. The shell filling is reflected by large maxima in the ionization energy for atomic numbers $2,10,18, \ldots$, corresponding to noble gas atoms $\mathrm{He}, \mathrm{Ne}, \mathrm{Ar}, \ldots$. The mid-shell levels are filled according to Hund's rules, i.e. by maximizing the total electron spin for half-filled orbitals (Chakraborty dan Apalkov 2003). In a quantum dot, atomic shells are described by a set of degenerated energy levels (Tarucha et al. 1998). Each degenerated set is filled by $2,6,12, \ldots$ electrons. The set number of $2,6,12, \ldots$ are known as "magic number" of electrons in the circularshaped 2-D harmonic potential confinement.

The Kondo effect is a universal phenomenon which is manifest itself when a localized state with a net spin couples with conduction electrons in the Fermi sea (Tamura et al. 2003). In a normal metal, a sea of conduction electrons and the localized moment of spin in a magnetic impurity are coupled by the effective exchange interaction, forming a spin-singlet bound state. The localized moment of spin is screened by the so-called "Kondo cloud" of conduction electrons as if the localized spin disappeared. This many-body effect is called the Kondo effect, i.e. a peculiar phenomenon in the temperature dependence of the resistance. The resistance decreased with temperature due to the reduction of phonon scatterings, but increased upon cooling to very low temperatures called Kondo temperature. The Kondo effect in quantum dots arises from the singlet coupling between a localized electron spin in a dot and a Fermi sea of conducting electrons in the source and drain (Tarucha et al. 2001). Tarucha et al. 
observe a strong Kondo effect when the spin singlet and triplet are degenerate by tuning the spin configuration out of two electrons as a function of magnetic field (Tarucha et al. 2001).

The physical characteristics of an artificial atom differ considerably from that of a real atom because of its size typically much greater than real atoms. The electron orbits do not simply scale with size because in quantum dots one can control their size. As it is made larger, the Coulomb energy arising from the repulsion between electron decreases because the average spacing between electrons increases. As the atomic size increases, the difference in the orbital energies decreases faster than the Coulomb energy. It shows that in large atom, the effects of electron-electron interaction are relatively more important than in small atom (Ashoori 1996). What is most exiting is that many of quantum phenomena in real atom can be easily observed in quantum dots by simply changing its size or shape (Kouwenhoven dan Marcus 1998). Because of its tunable size, some phenomena which only occur in hundreds Teslas in real atom, can occur in a range of a few Teslas inside the quantum dot. It is caused by the larger area of the dot can receive more quantum magnetic flux.

\section{A MODEL FOR SINGLE ELECTRON QUANTUM DOT}

This paper will consider a two-dimensional electron system in a semiconductor heterostructure created by $n$-doping the $\mathrm{AlGaAs}$ on GaAs. Figure 2 shows type of sample use in this analysis schematically.

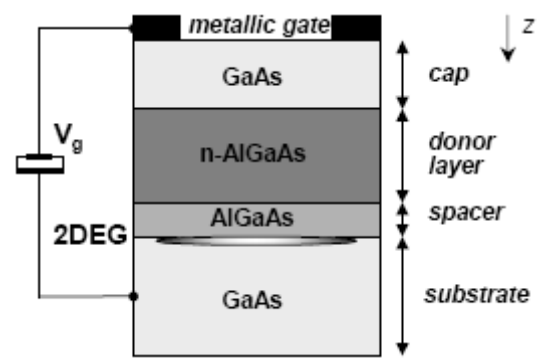

Figure 2. Heterostruacture AlGaAs-GaAs in the vertical cross-section. The 2DEG is localized

in the substrate near the interface with the spacer. Bias voltage Vg is applied between the gate and the substrate.

At negative gate voltages, the 2DEG is electrostatically squeezed from underneath the gate into the regions under the uncovered openings. As a result, the conduction band electrons will be confined in the $x-y$ plane, in addition to the vertical confinement at the heterointerface. By changing the lithography of the split gate, one can reduce the 2DEG to 1-D forming quantum wires and $0-\mathrm{D}$ forming quantum dots.

According to the similarity with real atom that electrons attracted to central potential i.e. nuclei attraction, this paper will consider bowl like potential confinement. The cross-section is a parabola so that electrons tend to move to bottom of the bowl so they exhibit oscillating in this bowl. The appropriate potential chosen here is harmonically oscillator potential $V=\frac{1}{2} m^{*} \omega_{0}^{2} r^{2}$, where $m^{*}$ is the effective mass of the electron, $\omega_{0}$ is harmonic oscillator frequency, and $r$ measures the distance from the centre of quantum dot. Starting with Hamiltonian system

$H=\frac{p^{2}}{2 m^{*}}+\frac{1}{2} m^{*} \omega_{0}^{2} r^{2}$

one can find Schrödinger equation in the polar coordinate system as

$$
-\frac{\hbar^{2}}{2 m^{*}}\left(\frac{\partial^{2}}{\partial r^{2}}+\frac{1}{r} \frac{\partial}{\partial r}+\frac{1}{r^{2}} \frac{\partial^{2}}{\partial \varphi^{2}}\right) \psi+\frac{1}{2} m^{*} \omega_{0}^{2} r^{2} \psi=E \psi
$$

Using variable separation methods, the wavefunction $\psi(r, \varphi)$ can be written as the product of a radial part $R(r)$ and an angular part $\Phi(\varphi)$

$\psi(r, \varphi)=R(r) \Phi(\varphi)$

Angular part of Schrödinger equation can be easily solved by $\Phi_{m}(\phi)=\frac{1}{\sqrt{2 \pi}} e^{-i m \phi}$, where $e^{-i m \phi}$ is an eigenfunction of $L_{z}$ with integer quantum number $m=0, \pm 1, \pm 2, \ldots$ as they satisfy boundary condition $\Phi(\varphi+2 \pi)=\Phi(\varphi)$. Then, radial part of Schrödinger equation is formulated by $\left[\frac{\partial^{2} R(r)}{\partial r^{2}}+\frac{1}{r} \frac{\partial R(r)}{\partial r}-\frac{m^{2}}{r^{2}} R(r)\right]+\left[k^{2}-\lambda^{2} r^{2}\right] R(r)=0$

with $k^{2}$ connected to the eigenvalue $E=\frac{\hbar^{2} k^{2}}{2 m^{*}}$, and $\lambda=\frac{m^{*} \omega_{0}}{\hbar}$ is a characteristic oscillator quantity.

By mean of a meaningful physical wavefunction namely $R(r)$ remains finite as $r \rightarrow 0$ and $\quad R(r) \rightarrow 0$ as $r \rightarrow \infty$, differential equation (5) in the neighbourhood of $r \approx 0$ at the lowest order 
$\frac{\partial^{2} R(r)}{\partial r^{2}}+\frac{1}{r} \frac{\partial R(r)}{\partial r}-\frac{m^{2}}{r^{2}} R(r)=0$

Because $R(r)$ must be finite around the origin, $R(r)$ can be write down as a Taylor expansion for $r \approx 0$ and the first term in the expansion has to be $R(r) \approx r^{s}$ with $S$ is an integer (a constant is not a solution of (6)). From the compatibility condition $s^{2}-m^{2}=0$, the value of $s=|m|$ must be chosen to keep $R(r)$ finite at the origin. By means of a similar argument, the asymptotic form of $R(r) \rightarrow 0$ as $r \rightarrow \infty$ can be formulated by $R(r) \approx \mathrm{e}^{-\lambda r^{2} / 2}$. Thus combining the two results above, the form of the true wavefunction of radial part is given by

$R(r)=r^{|m|} \mathrm{e}^{-\lambda r^{2} / 2} F(r)$

where $F(r)$ must tend to a non-zero constant value as $r \rightarrow 0$ and cannot diverge faster than $\mathrm{e}^{-\lambda r^{2} / 2}$ as $r \rightarrow \infty$. Inserting (7) into (5) give a result

$\frac{\mathrm{d}^{2} F(r)}{\mathrm{d} r^{2}}+\left[\frac{2|m|+1}{r}-2 \lambda r\right] \frac{\mathrm{d} F(r)}{\mathrm{d} r}-\left[2 \lambda(|m|+1)-k^{2}\right] F(r)=0$

Introducing a new dimensionless variable $x=\lambda r^{2}, \quad F(r)$ is transformed into Kummer equation

$x \frac{\mathrm{d}^{2} F(x)}{\mathrm{d} x^{2}}+[(|m|+1)-x] \frac{\mathrm{d} F(x)}{\mathrm{d} x}-\frac{1}{2}\left[(|m|+1)-\frac{k^{2}}{2 \lambda}\right] F(x)=0$

whose solution regular at $x=0$ is the Confluent Hypergeometric Series (Abramowits dan Stegun 1972).

$F(x)=M(a,|m|+1 ; x)$

where $a=\frac{1}{2}(|m|+1)-\frac{k^{2}}{4 \lambda}$. For large values of $x$,

this function would diverge as $\mathrm{e}^{x}$, thus preventing normalization. If, and only if $a=-n$ with $n=0,1,2, \ldots$ integer radial quantum number, the Hypergeometric Confluent Series becomes a polynomial and the wavefunction can be normalized. Then the solution of eq. (3) is given by

$\psi_{n m}(r, \varphi)=C_{n m} r^{|m|} \mathrm{e}^{-\lambda r^{2} / 2} M\left(-n,|m|+1 ; \lambda r^{2}\right) \mathrm{e}^{-i m \varphi}$

and

$E_{n m}=\hbar \omega_{0}(2 n+|m|+1)$

Here, $C_{n m}$ are normalization factors, $E_{n m}$ is the eigenvalue depending on the couple of radial and azimuthal quantum number $(n, m)$ and $\psi_{n m}$ are called Fock-Darwin states, after Fock and Darwin calculated eigenvalue of a particle in a harmonic potential and an external magnetic field independently (Fock 1928).

Figure 3 shows the shell structure of the single-particle energy spectrum and the associated degeneracies schematically.

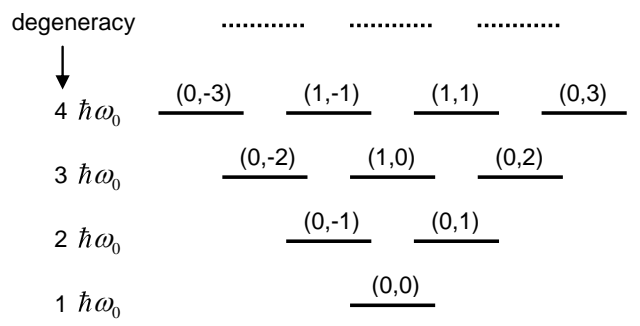

Figure 3. Shell structure of single-particle energy spectrum.

Each shell corresponds to the energy $\hbar \omega_{0}(N+1)$ where $N=2 n+|m|$ is fixed, and $(n, m)$ are the radial and azimuthal quantum numbers, respectively. The degeneracy of each shell (not taking account of spin) is $N+1$.

Since an electron can have spin-up or spindown, each energy level can be occupied by two electrons. The state $n=0, m=0$ occupied by two electrons with opposite spin. The next state can be occupied by 4 electrons, two electrons are in state $(0,1)$ and the others are in state $(0,-1)$. Finally, the second shell is fully filled by $N=6$ electrons. The third shell can be occupied by 6 electrons, each pair occupies the $(0,2),(0,-2)$ and $(1,0)$ state. So the third shell is fully filled by 12 electrons. These fully filled shells lead to the magic number of $N=2,6,12,20$ which are exactly similar to the experiment result done by Tarucha et al. 1996.

In order to calculate normalization factors $C_{n m}$ of equation (11), let consider the Confluent Hypergeometric Series. For special values of parameters, it reduces to simpler classes of orthogonal polynomials, in this case the Generalized Laguerre Polynomials. The connection is formulated by

$L_{n}^{|m|}(x)=\frac{(n+|m|) !}{n !|m| !} M(-n,|m|+1 ; x)$

and an expression for $L_{n}^{|m|}(x)$ is given by

$L_{n}^{|m|}(x)=\sum_{i=0}^{n}(-1)^{i} \frac{(n+|m|) !}{(n-i) !(|m|+i) ! i !} x^{i}$

with the first few polynomials can be write down as $\quad L_{0}^{0}(x)=1, \quad L_{0}^{1}(x)=1, \quad L_{0}^{2}(x)=1$, 
$L_{1}^{0}(x)=-x+1, \quad L_{0}^{3}(x)=1, \quad L_{1}^{1}(x)=-x+2, \ldots$.

Thus, the orthogonality relations give

$\int_{0}^{\infty} \mathrm{d} x \mathrm{e}^{-x} x^{|m|} L_{n}^{|m|}(x) L_{n^{\prime}}^{|m|}(x)=\delta_{n, n^{\prime}} \frac{(n+|m|) !}{n !}$

which combining of equation (13) and (15) make it possible to calculate normalization factors $C_{n m}$. Then, expression (11) for the properly normalized Fock-Darwin states turns into

$\psi_{n m}(r, \varphi)=\lambda^{(|m|+1) / 2} \sqrt{\frac{n !}{\pi(n+|m|) !}} r^{|m|} \mathrm{e}^{-\lambda r^{2} / 2} L_{n}^{|m|}\left(\lambda r^{2}\right) \mathrm{e}^{-i m \varphi}$

with few explicit formula are given below:

$\psi_{00}(r, \varphi)=\sqrt{\frac{\lambda}{\pi}} \mathrm{e}^{-\lambda r^{2} / 2}$

$\psi_{0 \pm 2}(r, \varphi)=\sqrt{\frac{\lambda}{2 \pi}} \lambda r^{2} \mathrm{e}^{-\lambda r^{2} / 2} \mathrm{e}^{\mp i 2 \varphi}$

$\psi_{0 \pm 1}(r, \varphi)=\frac{\lambda}{\sqrt{\pi}} r \mathrm{e}^{-\lambda r^{2} / 2} \mathrm{e}^{\mp i \varphi}$

$\psi_{10}(r, \varphi)=\sqrt{\frac{\lambda}{\pi}}\left(1-\lambda r^{2}\right) \mathrm{e}^{-\lambda r^{2} / 2}$

A few wavefunctions corresponding to the three lowest energy shells are plotted in Figure 4. The plot is along the $x$-axis, at $y=0$ and $\varphi(0, \pi)$ where all wavefunctions $\psi_{n m}$ are real along $x$-axis and depend only on the absolute value of $m$ while in general wavefunctions are complex, and the phase depends both on the value and sign of $m$. It has seen that the state $(0, \pm 1)$ changes sign under $x \rightarrow-x$ reflection (in general, under a reflection with respect to a plane containing the origin and the raxis), while the other states are symmetric.

\section{FOCK-DARWIN SPECTRUM IN AN EXTERNAL MAGNETIC FIELD}

It is interesting to observe the effect of magnetic field $\vec{B}$ on the atom-like properties. This paper will review the influence of an external magnetic field perpendicular to a $2 \mathrm{D}$ single electron polar harmonic potential. A magnetic field has a negligible effect on both Zeeman spin splitting, $g \mu_{B} \vec{B}$ which is only $\square 0.025 \mathrm{meVT}^{-1}$ in GaAs since $g_{\mathrm{GaAs}}=-0.44$ (Kouwenhoven et al. 2001), and the spin-orbit interaction. In this case, Hamiltonian system is

$H=\frac{1}{2 m^{*}}\left[\vec{p}-\frac{e}{c} \vec{A}(\vec{r})\right]^{2}+\frac{1}{2} m^{*} \omega_{0}^{2} r^{2}$

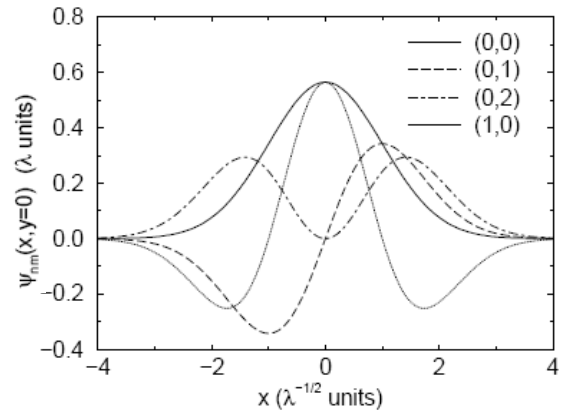

Figure 4. Plot of Fock-Darwin wavefunctions along the $x$-axis, with all $\psi_{n m}$ 's are real along this direction.

where $\vec{B}=B \hat{z}$ and vector potential $\vec{A}(\vec{r})$ is specified to be Gauge symmetric with $\vec{A}=\frac{1}{2} \vec{B} \times \vec{r}$. It's mean that the symmetric gauge is also a Coulomb gauge, i.e. $\nabla \cdot \vec{A}(\vec{r})=0$, and hence $[\hat{\vec{A}}(\vec{r}), \hat{\vec{p}}]=0$. Then, the term in bracket can be derived as

$\frac{1}{2 m^{*}}\left[\vec{p}-\frac{e}{c} \vec{A}(\vec{r})\right]^{2}=\frac{\vec{p}^{2}}{2 m^{*}}+\frac{e}{2 m^{*} c} \vec{p} \cdot(\vec{r} \times \vec{B})+\frac{e^{2} B^{2}}{8 m^{*} c^{2}} r^{2}$

By means of the triple product rule $\vec{A} \cdot \vec{B} \times \vec{C}=\vec{A} \times \vec{B} \cdot \vec{C}$ and defining the cyclotron frequency $\omega_{c}=|e| B / m^{*} c$, Hamiltonian system become $H=-\frac{\hbar^{2}}{2 m^{*}}\left(\frac{\partial^{2}}{\partial r^{2}}+\frac{1}{r} \frac{\partial}{\partial r}+\frac{1}{r^{2}} \frac{\partial^{2}}{\partial \varphi^{2}}\right)+\frac{\omega_{c}}{2} L_{z}+\left(\frac{m^{*} \omega_{c}^{2}}{8} r^{2}+\frac{m^{*} \omega_{0}^{2}}{2} r^{2}\right)$

By setting $\Omega^{2}=\omega_{0}^{2}+\frac{\omega_{c}^{2}}{4}$ and using the eigenvalues of $L_{z}=-m \hbar$, the same procedure has been used in previous section give a new energy egenvalues as

$$
\begin{aligned}
E_{n m} & =\hbar \Omega(2 n+|m|+1)-m \hbar \frac{\omega_{c}}{2} \\
& =\hbar \sqrt{\omega_{0}^{2}+\frac{1}{4} \omega_{c}^{2}}(2 n+|m|+1)-m \hbar \frac{\omega_{c}}{2}
\end{aligned}
$$

From the Fock-Darwin spectrum appears in (21), when $\omega_{c}>\omega_{0}$ electron will enters Landau regime where $E_{n}=\left(n+\frac{1}{2}\right) \hbar \omega_{c}$. In this region, the harmonic potential is smaller than the external magnetic field, so the electrons inside the dot behave as if they are free particles under the magnetic field. Thus, the Fock-Darwin spectrum can be seen in Figure 5 
(Kouwenhoven et al. 2001) as a few lowest eingenvalues $E_{n m}$ (in units of $\hbar \omega_{0}$ ) vs the magnetic field $B$ (expressed as $\omega_{c} / \omega_{0}$ ).

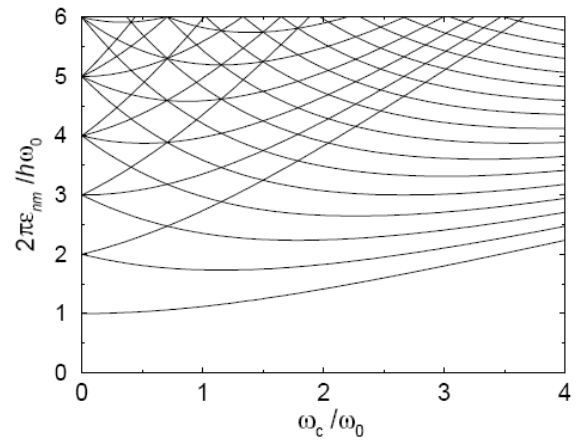

Figure 5. Plot of a few lowest eigenvalues $E_{n m}$ (in units of $\hbar \omega_{0}$ ) for a single particle parabolic confinement potential vs magnetic field (in units of $\left.\omega_{c} / \omega_{0}\right)$.

Figure 5 shows that as $B$ is increased, the levels labeled by the maximum value of $m$ go first downwards and then slightly upwards crossing all levels of lower shells but the ones corresponding to the maximum value of $m$ within one shell. In other word, when the external magnetic field is increased, the electron in the highest state can cross to a different state. In the limit $B \rightarrow \infty$, these lowest eigenvalues (i.e. those labeled by $(0, m)$, with $m>0)$ tend to an asymptotic form corresponding to the lowest Landau level. A second asymptote, with a steeper slope, corresponding to the first excited Landau level, accumulates a second bunch of Fock-Darwin states, as long as $B$ rises, and so on. This oscillation will end after the electron enters the Landau regime. Reference (Datta 1995) gives more detailed analysis.

\section{CONCLUSION}

Theoretical study of a single electron quantum dot has been reviewed in this paper. Analytical solutions show many of atomic-like phenomena observed in semiconductor quantum dots. Calculation of shell structure, FockDarwin state and the Fock-Darwin spectrum in an external magnetic field give a good agreement with experimental result.

\section{FURTHER RESEARCH}

Theoretical investigation on electronic structure of few electron quantum dots will be done using density functional theory approach based variational principle. The physics of quantum dot, e.g. thermodynamic properties of quantum dot and possibility of phase transition phenomena is an interesting subject to be studied in the next work.

\section{REFERENCES}

Abramowitz, M. dan Stegun, I.A. (1972), Handbook of Mathematical Functions, Dover, New York.

Ashoori, R.C. (1996), 'Electrons in Artificial Atoms', Nature, 379, p. 413.

Chakraborty, T. dan Apalkov, V.M. (2003), 'Quantum Cascade Transition in Nanostructures', Advanced in Physics, 52 (5), p. 455 .

Datta, S. (1995), Electronic Transport in Mesoscopic Systems, Cambridge University Press, Cambridge, Chapter 1.

Fock, V. (1928), 'Bemerkung zur Quantelung des Harmonischen Oszillators im Magnetfeld', Zeitschrift fur Physik, 47, p. 446.

Kouwenhoven, L.P. dan Marcus, C. (1998), 'Quantum dots', Physics World, June 1998.

Kouwenhoven, L.P., Austing, D.G., dan Tarucha, S. (2001), 'Few-electron Quantum Dot', Report on Progress in Physics, 64, p. 701.

Palmer, D.W. (2003), 'The SemiconductorsInformation: www.semiconductors.co.uk', accessed 17 April 2003.

Tamura, H., Shiraishi, K., dan Takayanagi, H. (2003), 'Quantum Dot Atoms, Molecules, dan Superlattices', in Quantum Dots and Nanowires edited by Bandyopadhyay, S., dan Nalwa, H.S., American Scientific Publisher, USA.

Tarucha, S., Austing, D.G., Honda, T., van der Hage, R.J., dan Kouwenhoven, L.P. (1996), 'Shell Filling and Spin Effect in a Few Electron Quantum Dot', Pbysical Review Letter, 77 (17), p. 3613.

Tarucha, S., Austing, D.G., Sasaki, S., Fujisawa, T., Tokura, Y., Elzerman, J.M., van der Wiel, W., de Franseschi, S., dan Kouwenhoven, L.P. (2001), 'Novel Kondo Anomaly in Quantum Dots', Materials Science and Engineering, B84, p. 10.

Tarucha, S., Honda, T., Austing, D.G., Tokura, Y., Muraki, K., Oosterkamp, T.H., Janssen, J.W., dan Kouwenhoven, L.P. (1998), 'Electronic States in Quantum Dot Atoms and Molecules', Physica, E3, p. 112.

Thouless, D.J. (1977), 'Maximum Metallic Resistance in Thin Film', Physical Review Letter, 39(18), p. 1167.

Diterima: 23 Maret 2007

Disetujui untuk diterbitkan: 14 Mei 2007 